\title{
Miedo, desarraigo y proyectos familiares en la población inmigrante venezolana en el Perú
}

\author{
Jerjes Loayza Javier \\ Universidad Nacional Mayor de San Marcos, Lima, Perú \\ mxerxeslj@gmail.com \\ Rubén Ticona Fernández Dávila \\ Universidad Ricardo Palma, Lima, Perú \\ ruben.ticona@urp.edu.pe
}

\begin{abstract}
RESUMEN
El artículo explora los miedos y temores que experimentan cotidianamente diversos inmigrantes venezolanos radicados en el Perú. Se ahonda en las dinámicas de aproximación entre peruanos y venezolanos, así como en sus consecuencias en el nivel intersubjetivo. Metodológicamente, se parte de entrevistas recolectadas en Ayacucho, Chiclayo, Cusco y principalmente en Lima. Fundamentalmente, se estudian las historias de vida de inmigrantes en situación de vulnerabilidad, ya sea sin trabajo, pidiendo ayuda económica en la calle o que haya vivenciado alguna experiencia difícil. Finalmente, se hilvanan las perspectivas a futuro que poseen identificando una serie de esperanzas que abrigan para sobrevivir a la explotación y discriminación de la cual serían objeto, aunque sin dejar de abordar los entramados esperanzadores en un país que, según se entiende, no deja de ser un importante destino migratorio.
\end{abstract}

Palabras Clave: Inmigración venezolana, desarraigo, familia, expectativas a futuro, xenofobia, explotación laboral

\section{Fear, estrangement and family projects in the immigrant Venezuelan population in Peru}

\begin{abstract}
This article analyzes the fears and concerns many Venezuelan immigrants living in Peru go through every day. It delves into the communication dynamics between Peruvians and Venezuelans, as well as into its consequences at an intersubjective level. Methodologically, it is based on data gathered from interviews in Ayacucho, Chiclayo, Cusco and mainly in Lima. Basically, the life histories of immigrants in vulnerable situation are analyzed, whether they are unemployed, asking for financial support on the street or having undergone any difficult experience. Finally, the expectations they hold on the future are joined together, identifying a series of hopes they embrace to survive to the exploitation and discrimination they would face. However, this study still addresses the encouraging frameworks in a country that, as it is understood, is still an important migration destination for them.
\end{abstract}

KeYwords: Venezuelan immigration, estrangement, family, future expectations, xenophobia, labor exploitation 


\section{Introducción}

El Perú ha presenciado, durante cuatro años a la actualidad, la llegada de más de 850 mil ciudadanos venezolanos, convirtiéndose en el segundo país, después de Colombia, con una mayor influencia migratoria sobre Venezuela ${ }^{1}$. Sin precedente a la vista ${ }^{2}$, se trata de un fenómeno que despertó, en un inicio, una exótica forma de visualizar a los recién llegados, para convertirse, poco a poco, en una percepción discriminatoria y xenófoba, llegando a los propios medios de comunicación que no dejaron de apelar a la incertidumbre y al miedo de la presencia de tal cantidad de migrantes. No solo se percibiría estos temores a nivel delictivo, sino por su impacto económico en el debilitado e informal esqueleto laboral peruano. Ello no solo ha ocurrido en Lima, sino en las regiones más representativas y pobladas del Perú, generando una serie de conflictos a nivel xenófobo en el trato cotidiano, patriarcal en el trato diferenciado hacia la mujer y laboral en la explotación que sufren debido a su condición inestable inmigrante ${ }^{3}$.

Partimos de la necesidad de estudiar un momento de suma trascendencia en el desarrollo demográfico, económico, político y cultural en el Perú, en tanto se van recreando numerosas formas de entender la vida cotidiana y la vida social, las cuales algunas veces se acercan para cimentarse y, otras veces, para repelarse y enfrentarse. Gracias a los testimonios obtenidos de la participación voluntaria de sus protagonistas, se identificarán los principales temores que albergan en su imaginario colectivo. A ello se le sumarán las expectativas de futuro que poseen y que, creemos, servirán de base fundamental para aminorar la incertidumbre de la cual son objeto en un país extraño y, muchas veces, violento con su presencia. La investigación parte de Lima, dada la cercanía de los autores con múltiples espacios de interacción inmigrante, y explora otras ciudades del país, tales como Chiclayo, Ayacucho y Cusco.

1 El número planteado se basa en las cifras de la Superintendencia Nacional de Migraciones, al año 2019 Recuperado de: https://migration.iom.int/system/tdf/reports/DTM_R6_VF.pdf?file=1\&type=node\&id=6670

2 Cabe destacar que el Perú, desde la conquista Europa acaecida en el siglo XVI, ha sido objeto de la llegada de múltiples inmigrantes con diversas intenciones, ya sean colonizadoras y políticamente impositivas (siglo XVI con los conquistadores españoles), acaso para el uso de mano de obra esclava o barata (africanos llegados durante los siglos XVII, XVIII y XIX; chinos culíes durante el siglo XIX; japoneses llegados a fines del siglo XIX e inicios del siglo XX) y, en general, la llegada de migrantes de diversas partes del mundo para encontrar un espacio acorde a las necesidades de la nueva familia fundante (italianos, irlandeses, alemanes, ingleses, polacos, entre otros principalmente durante fines del siglo XIX hasta la mitad del siglo XX).

3 Ver los estudios previos: Loayza Jerjes (2020) Inmigración venezolana y estigmatización laboral en el Perú y Loayza Jerjes (2020) Estigmatización laboral y patriarcalismo en la inmigración venezolana en el Perú. 


\section{Metodología}

Se presentan argumentos epistemológicos comprensivos, en tanto nos detenemos en el sentimiento y sensibilidad del inmigrante, el cual, de manera heurística, nos permite construir una serie de discursos y costumbres que vienen desarrollándose en el Perú. La recolección de datos se halla principalmente en Lima; también se utilizaron testimonios importantes en Ayacucho, Cusco y Chiclayo. La muestra elegida es de carácter voluntario y por conveniencia, debido a la dificultad de poder profundizar en la emotividad del inmigrante de carácter confesional, necesario para explorar con detenimiento en los miedos experimentados en el Perú. El caso de los testimonios pertenecientes a Cusco, Ayacucho, y Chiclayo fueron recolectados en el año 2019. En cuanto a los testimonios en Lima fueron brindados por diversos inmigrantes durante los ańos 2017-2019. Se recurrieron a tres técnicas: entrevistas semi estructuradas, entrevistas a profundidad e historias de vidas. Cabe destacar que se cumplió con los requerimientos éticos ${ }^{4}$ propios de temáticas en donde se vulneran los derechos de los protagonistas.

\section{Marco teórico}

La migración ha representado un problema de trascendencia mundial, derivándose a un enfrentamiento directo entre el norte y el sur, entre los países desarrollados y los países en vías de desarrollo. Formaría parte de una aceleración inédita que no se limita a una inestabilidad o incertidumbre, sino también "desamparos, desarraigos y miedos colectivos e individuales, pero fundamentalmente ha generado una desorientación histórica como colectividad" (Ticona, 2020, p. 161 ). En este difícil contexto, las explicaciones que tratan de entender las dimensiones de la migración como necesidad antes que como un acto de libre elección apuntan a una "creciente integración e interdependencia de países y mercados, por encima de las fronteras nacionales (lo cual generaría) exclusión, pobreza y desigualdad a nivel global” (Zabalo y Mesa, 2004, p. 5). El acto de inmigrar a un país en donde las diferencias culturales son o bien grandes o bien muy grandes, representa un verdadero reto para las poblaciones que se ven en la necesidad de hacerlo. Esta necesidad e inevitabilidad algunos la entienden como una especie de solución al declive de la población en edad activa, sin embargo, la emigración obedece a proyectos personales con determinados objetivos y no a comportamientos misioneros que intenten rejuvenecer poblaciones envejecidas, especialmente en Europa (Khader, 2004).

4 Se dejó en claro que se trataba de una investigación, así como del permiso expreso para la aplicación de una grabadora para, posteriormente, transcribir y, finalmente, destruir el audio requerido para la investigación. 
Emigrar para inmigrar genera esfuerzos enormes que atentan contra una determinada socialización, signos y basamentos históricos: implica experimentar identidades sometidas a mutaciones perennes, en tránsito, con la promesa de volver a casa, aunque "completar la historia, domesticar el circuito- se vuelve imposible (...) el lugar de partida y el punto de llegada no son inmutables ni seguros" (Chambers, 1994: 19). En Suráfrica, por ejemplo, Segatti (2004) identifica el modo en que el migrante mozambiqueño es empujado contra los márgenes de la informalidad. Sus entrevistados refieren sentimientos de persecución, generando traumas vinculados al desarraigo y la extrema pobreza. En el Perú Ansión, Mujica y Villacorta (2008) han estudiado este tema a nivel de migración interna, identificando los traumas o desarraigos que provocan las ausencias. Sin embargo, también identifican reacciones creativas, produciendo capacidades de resistencia y recuperación de la carencia para evitar caer en entrampamientos autodestructivos. En el ámbito de la inmigración internacional ello se vuelve aún más complejo dado que el desplazamiento ya no es, solamente, de un solo individuo, sino que "el grupo integrado por familias completas, que están dispuestas a padecer los infiernos de la travesía, antes que quedarse en sus lugares de origen a soportar las inclemencias de la falta de empleo y la pobreza" (Vázquez y Bocanegra, 2015, p. 31). En estos casos se fortalecen los lazos familiares, aunque se ven, también, amenazados por la inclemencia de las carencias y ausencias materiales.

Bajo dicha presión el migrante desarrollará, nos dicen Ansión, Mujica y Villacorta (2008) mecanismos de defensa y protección apelando no solo a la solidaridad entre los miembros de su familia, así como de sus connacionales, sino de instituciones no gubernamentales. Para los autores mencionados, la organización "no solo sirve para subsistir, sino también para ampliar las propias capacidades en otros espacios (incorporando inclusive) el recurso a la religiosidad como fuente de protección importante en la medida que da seguridad y sentido a las cosas que hacen los que permanecen" (Ansión, Mujica y Villacorta, 2008, p. 45). En este proceso la asimilación cultural es una posibilidad, en la medida que, plantea Tood (1996) el grupo inmigrado decida ingresar simbólicamente en una sociedad con la que se debería identificar. Para este autor la asimilación implica "un proceso antropológico cuyos principales actores son los inmigrantes y las capas populares (en la medida que) la interacción de esos grupos en las ciudades y en sus cinturones trae consigo cambios de costumbres y cierta frecuencia de matrimonios mixtos" (Tood, 1996, p. 352). A decir de Ávila, el inmigrante, para lograr integrarse, no experimentará un mero "trasplante" de "redes étnicas" originarias, sino un proceso de reinvención de nuevas redes, asistiendo al desarrollo de una nueva sociabilidad y cultura, es decir, a la reinvención de ego (Ávila, 2009). La inmigración, entonces, representaría un proceso inacabado de trayectorias constantes y de transformaciones continuas. La ambivalencia, la incertidumbre y el desarraigo se convertirían en persistentes acompañantes de viaje. 


\section{Resultados}

\section{Miedos y desarraigo}

Aun cuando se inmigre, el peligro de ser extraditado será patente: "a las cinco de la mañana nos cayó migración (...) al primo de mi mujer que está vendiendo caramelos por ahí, migración le cayó primero a él. Nosotros estábamos tirados allá ayer y a la siguiente nos cayó migración mientras descansábamos, pero les explicamos que tenemos papeles, todo fino". ${ }^{5}$ A ello se le suma la situación estructural de un país como el Perú que deberán heredar para sobrevivir, aun sin entender del todo la razón de las asimetrías sociales, económicas y políticas existentes. A pesar de ello, lo más importante es comer: "Al menos comemos aquí, allá se come una solo vez, si no tienes cómo"6. La alimentación juega un papel fundamental en la vida de los hijos que se desea mantener. Son repetidos los testimonios que inciden en la oportunidad de comer más, y en mayor calidad, a diferencia de lo que comían en Venezuela:

Hombre: Quizás no, los que pasa es que allá por la familia comemos un plato de comida, no todo el tiempo.

Mujer: Por lo menos no carne, no leche, no azúcar. Antes nosotros comíamos el plátano, que le llaman bellaco, lo comíamos sancochado o frito con un apanadito pero nada de carne, pollo, leche o mermelada. Ya por lo menos (su hija) en su escuela la merienda ya no le mandaba porque no tenía que ponerle ${ }^{7}$

Comprar insumos básicos para la crianza, por ejemplo, termina siendo imposible: "Tengo dos hijos y utilizan pañal. Ahorita la situación está fuerte porque un paquete

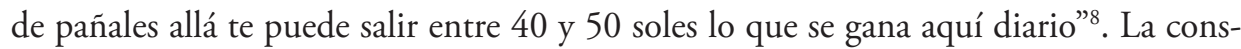
trucción de una familia tendrá costos elevados, obligando a los progenitores a esforzarse más allá de lo sospechado. Una mujer inmigrante en Chiclayo relata la necesidad de inmigrar: "venirme para aquí a otra vida, fuera de Venezuela, porque en Venezuela no se puede hacer nada y segundo bueno estoy por aquí porque quiero luchar para darles co-

5 Entrevista aplicada en Cusco en octubre del 2019. Hombre de 24 años. Llegó a Perú de la ciudad de Margarita en octubre del 2018. Es casado y tiene un niño de 4 ańos. Toda la familia vive en Perú. Trabaja vendiendo chutepes en Cusco

6 Entrevista aplicada en Cusco en octubre del 2019. Hombre de 24 años. Llegó a Perú de la ciudad de Margarita en octubre del 2018. Es casado y tiene un niño de 4 ańos. Toda la familia vive en Perú. Trabaja vendiendo chupetes en Cusco

7 Entrevista a un hombre de 36 y una mujer de 30 ańos, ambos casados. Junto a ellos dos de sus menores hijos una de semanas de nacida y, la otra, de 6 años de edad. Son naturales de Caracas. Aunque llevaban un ańo y medio en Lima, habían llegado aquel mismo día a Ayacucho.

8 Hombre de 30 años, natural de Guárico, Venezuela. Lleva dos años en Perú y recientemente llegado a Ayacucho. Se encontraba pidiendo limosna en la calle. Entrevista de octubre del 2019. 
sas a mis hijos, poco a poco". ${ }^{9}$ Inclusive los hijos serían capaces de avizorar la diferencia, identificando en Perú una mejor prosperidad: Bien, están felices (sus hijos) Les gusta el Perú, dicen que por aquí se ven más cosas, que los negocios están llenos de cosas y que por allá (Venezuela) nada, hasta hay que hacer cola pa' comprar ${ }^{10}$. Aun cuando todo sea diferente: "Las playas de Venezuela, no son como las de acá... es diferente; la comida es excelente, pero es diferente a la de Venezuela" ${ }^{11}$ Lo importante es vivir, sea aquí o allá, y comer, aun cuando el sabor no sea nada familiar.

Sin embargo, el precio para vivir, trabajar y trascender de su situación más difícil, se deberán soportar una serie de abusos, en particular y en muy especial grado, en el ámbito laboral. Un migrante en Chiclayo responde a la posibilidad de haber sido explotado:

Depende de la persona y el sitio, porque tú vas acá en provincia y es diferente a la capital. La gente es más humana en provincia que en la capital. Por ejemplo, antes yo trabajaba con una distribuidora de carne, y era muy explotador, podías trabajar ahí más de 12 horas y él te pagaba igual sueldo mínimo y a veces tú ni descansabas, la gritadera y uno se cansa de esas cuestiones, ya uno no se puede quejar mucho (...) Entre más me emplee no pienso mucho en mi familia, no me aflijo tanto ${ }^{12}$.

Aunque la explotación de la que se es objeto sea muy cruda, es difícil resistirse en tanto la situación es muy urgente. Además, ser extranjero implica una vulnerabilidad que dificulta la exigencia de derechos laborales. Finalmente, el trabajo arranca la nostalgia de sentirse solo y lejos de su propia familia. Es importante discutir este aspecto dado que la familia termina siendo, sostienen Ansión, Mujica y Villacorta (2008) la institución más pequeña que construye alianzas, es decir, vínculos sociales. Tal como lo demuestran los testimonios citados, permite afrontar "los retos de la producción (para la subsistencia) y de la reproducción (para la sucesión de generaciones), reconstruyendo el tejido social y el modo de estar en sociedad (la cultura), de modo que no puede reducirse a sus meras funciones materiales" (Ansión, Mujica y Villacorta, 2008, p. 37).

En este sentido, y siendo tan importante la protección de la familia transnacional en situaciones de separación, para Kline es importante que entre ambos países se fortalezcan proceso que posibiliten procedimientos de reunificación dado que "las familias deben tener el derecho, en la mayoría de los casos, de decidir cuál es el interés superior

9 Entrevista aplicada en la ciudad de Chiclayo en setiembre de 2019. Mujer de 26 ańos natural de Aragua. Tenía 2 semanas de haber llegado a Chiclayo. Vive con uno de sus hijos de 8 años y el otro menor aún vive en Venezuela.

10 Entrevista aplicada en la ciudad de Chiclayo en setiembre de 2019. Mujer de 26 años natural de Aragua. Tenía 2 semanas de haber llegado a Chiclayo. Vive con uno de sus hijos de 8 años y el otro menor aún vive en Venezuela.

11 Entrevista aplicada en Puerto Eten, Chiclayo en setiembre de 2019. Hombre de 23 ańos de Mérida. Vive con toda su familia en Perú. Trabaja como mesero en un restaurante de Puerto Etén.

12 Entrevista aplicada en Zaña, Chiclayo, Hombre de 32 años. Trabaja montando canchas de gras artificial. Vive en Chiclayo desde noviembre del 2019. 
de su familia y cómo es que quieren seguir adelante en la luz de interrupción a la vida familiar por políticas y prácticas migratorias" (Kline, 2015, p. 155).

Poco a poco se identifican las bases de un abuso frecuente en el ámbito peruano. Inclusive la ola de xenofobia producto de una incidencia negativa muy impactante en medios de comunicación debido a una serie de crímenes protagonizados por inmigrantes venezolanos: "Sí, estábamos viviendo en Lima, yo estaba trabajando pero por el tema de los venezolanos que han cometido crímenes, me botaron del trabajo (...) Simplemente por lo que se ve ahora, por la xenofobia" ${ }^{13}$ Un inmigrante en Ayacucho, como parte de las experiencias negativas halladas, se lamenta al no tener trabajo: "(Trabajaba) en el Callao, en el puerto. Duré seis meses, el señor muy amigo, pero se acabó la chamba. Entonces me dijo que fuera a descansar, más o menos, estoy sobreviviendo ahorita como quien dice". ${ }^{14}$ Sobrevivir en un país distinto con personas y culturas diferentes, conlleva a una contingencia muy violenta: "No, hasta ahora no, no he salido mucho. Estaba así puro caminado, ahorita que estoy así porque tengo que comer. (Las personas) No (apoyan) mucho, es lo que tengo estoy completando treinta soles, a ver si compro una bolsita de caramelos". ${ }^{15}$

Se debe entender que la búsqueda de apoyo, algo constante en los testimonios que comparten múltiples inmigrantes en este y otros estudios, obedece la necesidad de construir redes que funcionen ante determinados momentos de "temor o incertidumbre para buscar estabilidad y seguridad, o en épocas de carencias físicas y materiales, o cuando las personas han necesitado alimento o habitación, la red social y, particularmente, la función de la red social siempre ha sido la estructura desde la cual se ha apoyado socialmente para solventar necesidades inmediatas" (Rivera y Valdez, 2015, pp. 250-251). Sin embargo, los insultos y enfrentamientos callejeros, como producto de la xenofobia arraigada en la sociedad peruana hacia la población inmigrante venezolana, se convertiría en una constante lucha que deberán librar sus víctimas, con la necesidad de callar y soportar para evitar todo tipo de complicaciones dada su estadía migrante en un país sin garantía alguna para dicha condición.

Así, una mujer en Cusco dice: "(Lo peor son) los insultos que me dan las personas en la calle, en su mayoría mujeres. (No responde a los insultos) Porque no podemos faltarles el respeto a las personas mayores"16. Como se verá en otros testimonios, la dificultad de enfrentar las actitudes negativas de los demás hacia uno se ven descompen-

13 Entrevista a un hombre de 36 y una mujer de 30 ańos, ambos casados. Junto a ellos dos de sus menores hijos una de semanas de nacida y, la otra, de 6 años de edad. Son naturales de Caracas. Aunque llevaban un año y medio en Lima, habían llegado aquel mismo día a Ayacucho.

14 Hombre de 30 años, natural de Guárico, Venezuela. Lleva dos años en Perú y recientemente llegado a Ayacucho. Se encontraba pidiendo limosna en la calle. Entrevista de octubre del 2019.

15 Hombre de 30 ańos, natural de Guárico, Venezuela. Lleva dos años en Perú y recientemente llegado a Ayacucho. Se encontraba pidiendo limosna en la calle. Entrevista de octubre del 2019.

16 Entrevista realizada en Cusco con una mujer con una niña en los brazos vendiendo caramelos. La mujer tenía aproximadamente 30 años. 
sadas por ser inmigrante. Uno de ellos oye, a veces dirigido a él y otras a la comunidad de la cual es parte, insultos que preferirá, acaso por la imposibilidad de actuar de otro modo, soportar y olvidar:

Acá en el trabajo ahora últimamente un socio del club me dijo: "veneco de mierda” en una situación de juego. Yo estaba de árbitro y no le pareció uno, aunque luego me pidió disculpas, pero igual queda el insulto. También algunas veces comentarios en la calle cuando voy pasando hacia los venezolanos. Hace meses estaba yendo hacia la avenida Wilson y por ahí los que estaban en el carro les gritaban a ciertos venezolanos que son unos secuestradores, choros y cosas así. ${ }^{17}$

Los comentarios negativos hacia los comportamientos xenófobos del limeño promedio son repetitivos. Un hombre en Ayacucho supone que los problemas suscitados han provocado en la opinión publica un difícil ambiente de coexistencia: "No, allá (Lima) ha sido un poco difícil con todos los problemas que han pasado (...) no nos apoyan (...) también por la cantidad de papeles que pedían pues. Tú entiendes". ${ }^{18}$ Sobre la diferencia de trato entre limeños y el resto del Perú es algo repetitivo en diferentes testimonios: "En Piura la gente es más cerrada, en Trujillo la gente un amor, he viajado poco, pero me ha gustado siempre, te ofrecen ayuda, pero en Lima todo el mundo está pendiente del dinero, pero siempre hay alguien que tiene un gesto contigo (...) por ejemplo no tienes 0.10 centavos para completar algo y no te lo venden" ${ }^{19}$. A su vez, los insultos se mezclan con dinámicas patriarcales que humillan a la mujer, sin lugar alguno a poder tener defensa a tanta violencia callejera. Dos inmigrantes, que además eran pareja de novios, nos relatan su desgracia:

Hombre: A ella le gritan cosas diferentes.

Mujer: Las mujeres que se meten conmigo dicen «eres quita marido» o que busque trabajo a la mala gana. Lo gritan hombres, mujeres, todos, hasta las señoras mayores. Hombre: hubo un señor que ella le pidió una colaboración y prácticamente le dijo que se prostituyera.

Mujer: Le dije que no, y o sea me puse nerviosa y ahí fue cuando llego él (su pareja).

17 Entrevista realizada en noviembre del 2019 en la ciudad de Lima a un hombre de 35 años proveniente de Monagas. Llegó hace 1 ańo y 7 meses y trabajaba como recogedor de pelotas en un club de tenis.

18 Entrevista aplicada en setiembre del 2019 en la ciudad de Ayacucho. Hombre de 23 ańos natural de Anzoátegui. Contaba con dos meses en Perú y dos días en Ayacucho.

19 Entrevista realizada en el mes de mayo del año 2018 en la ciudad de Lima a un hombre de 24 años proveniente de Caracas. 
Hombre: no (le hice nada, solo) defenderla pues, porque a una mujer se le respeta, no se fue a nada físico porque había mucha gente y la verdad yo no estoy para eso, porque yo no vine para crear chongo entiendes ${ }^{20}$

Como se ve es muy difícil enfrentar los insultos o los agravios, en tanto se es extranjero en un país ajeno, vulnerando la posible capacidad de respuesta. Frente a este dilema algunos inmigrantes prefieren no ser considerados como extranjeros y esconderse, en lo posible de las malas miradas o los prejuicios. Un entrevistado en Lima asegura no haber tenido problemas, aunque ello implique invisibilizar su propia naturaleza extranjera: "Hasta ahora no he tenido problemas con peruanos porque trato de pasar desapercibido (...) Bueno siempre he sido muy amable y decente, pero claro con mi acento saben que uno no es de acá. Empiezan a mirarte mal o feo y lo que hago es ignorarlos"21.

\section{Presente, futuro y destino inmigrante}

¿Qué ocurrirá con él y la inmigrante que decide salir de su país? ¿Volverá a Venezuela en algún tiempo? ¿Decidirá seguir migrante hacia otros países? ¿Hay algún plan trazado o improvisarán de acuerdo con las circunstancias? Las respuestas obtenidas demuestran una clara ruta contingente. Trayectorias dubitativas e inseguras. Espacios en vías de construcción que se definen de acuerdo con su imaginación creatividad y resistencia. Uno de ellos tiene muy claro lo que desea: "Te hablo clarito, yo quiero sacar mi carnet de extranjería y ver si me da chance el año que viene, pues, si puedo tener una oportunidad aquí, a través de la situación que está pasando es difícil que pueda traer mi familia por acá, igual que en Venezuela, espero que se solucione las cosas por allá para quedarme porque ando prácticamente solo”. ${ }^{22}$ En efecto, llevar a cabo un plan implica una enorme voluntad dada las dificultades emocionales que transcurren en cada uno.

En palabras de Chávez, Yeraldi y Márquez (2015) el retorno a la comunidad de origen tendría repercusiones positivas y, a la vez, negativas. No todos los retornados poseen las mismas oportunidades al no poseer las mismas condiciones por las que tuvieron que emigrar. Unos tendrán recursos económicos pudiendo emprender negocios y pequeñas empresas, pero otros "simplemente se volverán a encontrar con su vieja historia y empezar de nuevo, peor que como cuando se fueron por su reinserción social" (Chávez, Yeraldi y Márquez, 2015, p. 73). Por ello ciertos testimonios no nos hablan aun en la

20 Entrevista aplicada en setiembre del 2019 en la ciudad de Chiclayo a una pareja de novios que provenían de Maracay. Ambos tienen 22 años. Llegaron 11 días antes de la entrevista.

21 Entrevista realizada en noviembre del 2019 en la ciudad de Lima a un hombre de 39 años proveniente de Barquisimeto. Llegó hace 1 ańo y 7 mese sy trabajaba como recogedor de pelotas en un club de tenis.

22 Hombre de 30 ańos, natural de Guárico, Venezuela. Lleva dos ańos en Perú y recientemente llegado a Ayacucho. Se encontraba pidiendo limosna en la calle. Entrevista de octubre del 2019. 
posibilidad de retornar, sino en seguir explorando otras posibilidades, estudiar, viajar y vivir. Ello en contradicción con el proceso globalizador que tiende a "reducir los espacios de lo posible para la mayoría de los pueblos de Sur, incentivando (directa e indirectamente) mayores migraciones hacia los países industrializados, a la vez que se incrementa la penalización y la respuesta autoritaria a estas migraciones" (Guevara, 2004, p. 185). Un entrevistado parte de la incertidumbre para plantearse, con suma expectativa, nuevos retos en su vida profesional:

No, la verdad no sé qué hacer con mi vida. Ahorita estoy trabajando nuevamente en lo mío, que es la música armando mi curriculum, quiero estudiar y terminar mis estudios. Me gustaría el conservatorio no sé (...) estudiar en el Perú si me gustaría, en una orquesta y así. Antes de tener este trabajo como profesor de música, pensé irme a Chile o Argentina, estoy viendo y no sé si quiero empezar de cero nuevamente. Perú es bonito, pero no sé qué hay al otro lado del mundo. ${ }^{23}$

De esta manera el futuro es contingente y el destino, a veces, debe remitirse a una posibilidad, aun cuando se tenga muchos deseos: "Vamos a ver, porque nuestro plan era pasar a Chile porque tenemos conocidos por allá, estamos viendo, vamos a ver, porque el problema para cruzar a Chile es que hay un problema con los papeles". ${ }^{24} \mathrm{~A}$ la larga, el destino a elegir dependerá, en la medida que sea posible, de encontrar mejores y mayores ofertas: "Bueno si sale un trabajo que le puedan pagar a uno, se puede quedar, sino tenemos que buscar otro lugar". ${ }^{25}$ No importa el lugar, lo importante es sobrevivir.

\section{Conclusiones}

La migración representa un problema con múltiples aristas, que ha degenerado en una carga de la cual nadie quiere ocuparse, haciendo del inmigrante un perseguido, discriminado y estigmatizado social. Tal como lo establece la teoría y los antecedentes, el proceso globalizador ha promovido la desigualdad a nivel mundial, provocando la necesidad de inmigrar, no como opción sino como obligación, con el objeto de sobrevivir junto a la familia, ya sea trasnacional o enteramente inmigrante. Los testimonios recolectados en diferentes departamentos del Perú, dan cuenta de la crudeza con que debe afrontar el inmigrante común la vida cotidiana. El miedo a ser excluidos, a no recibir socorro alguno o a ser menospreciados con insultos xenófobos, es parte del des-

23 Entrevista realizada en el mes de mayo del año 2018 en la ciudad de Lima a un hombre de 24 años proveniente de Caracas.

24 Entrevista a un hombre de 36 y una mujer de 30 ańos, ambos casados. Junto a ellos dos de sus menores hijos una de semanas de nacida y, la otra, de 6 años. Son naturales de Caracas. Aunque llevaban un ańo y medio en Lima, habían llegado aquel mismo día a Ayacucho.

25 Entrevista realizada en Cusco con una mujer con una nińa en los brazos vendiendo caramelos. La mujer tenía aproximadamente 30 ańos. 
arraigo que deben experimentar debido a la vulnerabilidad que caracteriza su condición migrante. La presencia de su pasado en Venezuela, si bien es patente, queda rezagado frente a la urgencia de perseguir nuevos horizontes.

Las fracturas emocionales que atraviesan en el vaivén migrante les obligarían a subordinarse a lógicas culturales que se imponen en el Perú: actitudes patriarcales, explotación laboral, informalidad económica, discriminación cotidiana, entre otras. Sin embargo, tales sensaciones se ven disminuidas por una perspectiva de futuro posible, puesto que, ante el destino errante e incierto, el inmigrante venezolano prefiere reivindicar su experiencia a través de las buenas personas y de los momentos más gratificantes, convenciéndose de que no todo es malo y que, a su vez, todo es posible. Si bien el Perú demuestra ser una sociedad sumamente asimétrica, no desfallecerán y negociarán, aun poniendo en juego sus propias emociones, en beneficio de sus familias y de su propio futuro. Poco a poco el camino va forjándose como uno posible, en donde el esfuerzo, la tolerancia y el amor por la familia, es capaz de alcanzar los horizontes sońados, sea en Perú o en cualquier otro país.

\section{Referencias}

Ansión. J, Mujica. L y Villacorta. A (2008). Los que no se quedan: familias de emigrados de un distrito de Lima. Federación Internacional de Universidades Católicas.

Ávila, J. (2009). Redes personales de africanos y latinoamericanos en Cataluña, España. Análisis reticular e integración y cambio. En Tipshe, № 7, pp. 9-38.

Chambers, I. (1994). Migración, cultura, identidad. Buenos Aires: Amorrortu editores.

Chávez, J, Yeraldi, G. y Márquez, A. (2015). Migración internacional y de retorno: desde Estados Unidos hacia Nayarit: desafíos y potencialidades. En Gloria Ciria y Alvaro Bracamonte, coordinadores. Crisis económica y politica antiinmigrante: efectos en familias mixtas en Estados Unidos y México. Hermosillo, Sonora: El colegio Sonora, pp. (47-84).

Guevara, J. (2004). Migraciones bolivianas en el contexto de la globalización. En Alternativa Sur, Vol.III, pp. 171-187.

Kline, V. (2015). Separación familiar por las políticas y prácticas migratorias de los Estados Unidos de América y su impacto en México. En Gloria Ciria y Alvaro Bracamonte, coordinadores. Crisis económica y politica antiinmigrante: efectos en familias mixtas en Estados Unidos y México. Hermosillo, Sonora: El colegio Sonora, pp. 145-156.

Loayza, J. (2020). Inmigración venezolana y estigmatización laboral en el Perú. En Investigaciones Sociales, 23(43), 179-192.

Loayza, J. (2020). Estigmatización laboral y patriarcalismo en la inmigración venezolana en el Perú. En Scientia, 22(22), 79-94. 
Rivera, O. y Valdez, G. (2015). Las redes sociales en el proceso migratorio de los menores: un análisis contemporáneo desde la movilidad, migración y retorno. En Gloria Ciria y Alvaro Bracamonte, coordinadores. Crisis económica y política antiinmigrante: efectos en familias mixtas en Estados Unidos y México. Hermosillo, Sonora: El colegio Sonora, pp. 247-280.

Sabalo, P. y Mesa, M. (2004). Génesis y desafíos de las migraciones internacionales: perspectivas del sur. En Alternativa Sur, Vol.III, pp. 7-15.

Segatti, A. (2004). Los refugiados mozambiqueños en Suráfrica: ¿Repartición voluntaria o devolución encubierta? Alternativa Sur, Vol.III, pp. 105-121.

Ticona, Rubén (2020). Extractivismo y la crisis civilizatoria. En PLURIVERSIDAD, (4), 151-164.

Tood, E. (1996). El destino de los inmigrantes: asimilación y segregación en las democracias occidentales. Barcelona: Tusquets Editores.

Vázquez, M. y Bocanegra, C. (2015). El proceso de migración de México hacia Estados Unidos. Comportamiento y características. En Gloria Ciria y Alvaro Bracamonte, coordinadores. Crisis económica y politica antiinmigrante: efectos en familias mixtas en Estados Unidos y México. Hermosillo, Sonora: El colegio Sonora. 